\title{
N- FOURIER SERIES EQUATIONS INVOLVING JACOBI POLYNOMIALS OF DIFFERENT INDICES
}

\author{
Indu Shukla \\ ${ }^{1}$ Research scholar, Department of Physical science, \\ M.G.C.G.V.,Chitrakoot, \\ Satna, M.P.(India)
}

\author{
Uday singh \\ Department of Mathematics and Computer science, \\ R.D. University \\ ,Jabalpur, M.P.(India)
}

\begin{abstract}
In this paper, we have considered the N-Fourier series equations involving Jacobi polynomials of different indices of the first and second kind and solved the two sets of series equations.
\end{abstract}

Keywords: Integral equation; Series equation; Fourier series; Integral theorems; Jacobi polynomials.

\section{INTRODUCTION}

Dwivedi and Trivedi [2] Considered quadruple series equations involving Jacobi polynomials of the same indices which are orthogonal to the weight function $(1-x)^{\alpha-\beta} x^{\beta-1}$ in the interval $(0,1)$ Szego [9] later on standardized the notation

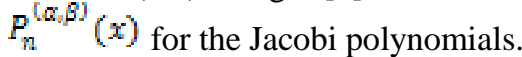

Askey [1] remarked that the dual series equations involving Jacobi polynomial of different indices cannot be solved. Later on Dwivedi and Gupta [4] obtained the solution of such quadruple series equations which include dual and triple series as particular cases. If we review the literature then we observe that the existing solutions on series equations are derived only from dual to six Fourier series equations, no further generalizations are available till date. This tempted us to find the solution of n-Fourier series equations involving Jacobi polynomials of different indices and in this paper we have obtained certain results. By considering the special values of $n$ $=2,3,4,5,6$ we shall be able to derive solutions of dual, triple,quadruple,5-tuple and 6-tuple Fourier series equations involvingjacobi polynomials of different indices[5], [6].

\section{N- SERIES EQUATIONS OF THE FIRST KIND}

1) N-series equations of the first kind involving jacobi polynomials of different indices are as follows :

$$
\sum_{m=0}^{m e} \frac{A_{m} P_{m}^{[(\alpha, \beta)}(x)}{(\beta+1)_{m} r(\alpha-\mu+m+1)}=f_{i}(x), \quad a_{i-1}<x<a_{i}
$$

$$
\begin{aligned}
& \text { where, } \mathrm{i}=1,3,5, \ldots \ldots, \mathrm{n}-1 \text { and } a_{0}=0 . \\
& \sum_{m=0}^{\infty} \frac{A_{m} P_{m}^{\left(\alpha+\beta-\varepsilon_{b}\right.} \omega_{(x)}}{(\alpha+\beta-\alpha+1)_{m} r(\beta+\mu+m+1)}=f_{j}(x), \quad a_{\mathrm{i}-1}<x<a_{\mathrm{i}}
\end{aligned}
$$

where, $\mathrm{j}=2,4,6, \ldots \ldots n$ and $a_{n}=\infty$

Here $\mathrm{n}$ is taken as an even number. If $\mathrm{n}$ is odd then the equations will be

$$
\begin{aligned}
& \sum_{m=0}^{m} \frac{B_{m} P_{m}^{[\alpha, \beta y}(\alpha)}{(\beta+1)_{m} r(\alpha-\mu+m+1)}=f_{i}(x), a_{i-1}<x<a_{\mathrm{i}} \\
& \text { where, } \mathrm{i}=1,3,5, \ldots \ldots \mathrm{n} \text { and } a_{0}=0 \text {. } \\
& \sum_{m=0}^{\infty} \frac{B_{m} P_{m}^{\left(\alpha+\beta-B_{0}\right.} \omega_{(x)}}{(\alpha+\beta-\sigma+1)_{m} r(\beta+\mu+m+1)}=f_{j}(x), \quad a_{j-1}<x<a_{j}
\end{aligned}
$$

2) N-series equations of the second kind

$\mathrm{N}$-series equations of the second kind involving jacobi polynomials of different indices are as follows :

$$
\begin{aligned}
& \sum_{m=0}^{\infty} \frac{D_{m} P_{m}^{(\alpha+\beta)-\varepsilon_{i}} \mathbb{E}_{(\alpha)}(\alpha+\beta-\sigma+1)_{m} \Gamma(\beta+\mu+m+1)}{(\alpha+\beta)}=g_{i}(x), a_{i-1}<x<a_{i} \\
& \text { where, } \mathrm{i}=1,3,5, \ldots \ldots \mathrm{n}-1 \text { and } a_{0}=0 \\
& \sum_{m=0}^{m} \frac{D_{m} P_{m}^{[\alpha, \beta]}(x)}{(\beta+1)_{m} r(\alpha-\mu+m+1)}=g_{j}(x), \quad a_{j-1}<x<a_{j} \\
& \text { where, } \mathrm{j}=2,4,6, \ldots \ldots \mathrm{n} \text {. }
\end{aligned}
$$

Here also $\mathrm{n}$ is taken as an even number. If $\mathrm{n}$ is odd then the equations will be

$$
\begin{aligned}
& \sum_{m=0}^{\infty} \frac{E_{m} P_{m}^{\left(\alpha+\beta-E_{0}\right.} \mathscr{E}_{([)}(x)}{(\alpha+\beta-\sigma+1)_{m} \Gamma(\beta+\mu+m+1)}=g_{i}(x), a_{i-1}<x<a_{i} \\
& \text { where, }{ }^{\mathrm{i}}=1,3,5, \ldots \ldots \mathrm{n} \text { and } a_{0}=0 \text {. } \\
& \sum_{m=0}^{m} \frac{E_{m} P_{m}^{[\alpha, \beta]}(x)}{(\beta+1)_{m} r(\alpha-\mu+m+1)}=g_{j}(x), \quad a_{j-1}<x<a_{j}
\end{aligned}
$$$$
\text { where, } \mathrm{j}=2,4,6, \ldots \ldots, \mathrm{n}-1 \text {. }
$$

Also $c>0,1$ is an arbitrary non- negative integer. $f_{i}(x), g_{i}(x)$, where $\mathrm{i}=1,3,5, \ldots, \mathrm{n}-1$ and $f_{j}(x), g_{j}(x)$ where $\mathrm{j}=2,4,6, \ldots \ldots n$ are $\mathrm{n}$ prescribed functions. $A_{m}, B_{m}, D_{m}$ and $E_{m}$ are unknown coefficients, are determined and the parameters $\alpha_{v} \beta, v, \delta, \lambda$ satisfy the conditions $\delta-\mu-\beta>0$,

$\alpha>-1, \beta>-1, \delta>-1, \alpha+\beta-\delta>-1$. Here we solve only equations (1),(2)of first kind and equations (5),(6) of the first kind and equations (7),(8) of the second kind will follow easily.

\section{PRELIMINARY RESULTS}

In the course of analysis, we shall use the following results:

(i) The orthogonality relation for the Jacobi polynomials

where, $\mathrm{j}=2,4,6, \ldots \ldots \mathrm{n}-1$. 
$\int_{-1}^{1} P_{n}^{[\alpha, \beta]}(x) P_{m}^{[\alpha, \beta]}(x)(1-x)^{\alpha}(1+x)^{\beta} d x=$ $2^{m+\beta+1} \Gamma(n+\alpha+1) \Gamma(n+\beta+1) a_{n}^{m}$

$(2 n+\alpha+\beta+1) \Gamma(n+1) \Gamma(n+\alpha+\beta+1)$

where $^{\delta_{n}^{m}}$ is the Kronecker delta,

(ii) The series

$$
\begin{aligned}
& S(r, x)=\sum_{n=0}^{m} \frac{(\alpha+\beta-\delta+1)_{n} \Gamma(\beta+\mu+n+1)(2 n+\alpha+\beta+1) \Gamma(n+1) \Gamma(n+\alpha+\beta+1)}{2^{\alpha+\beta+1} \Gamma(n+\alpha+\beta-\delta+1) \Gamma(n+\delta+1)(\beta+1)_{n} \Gamma(n+1+\alpha-\mu)}
\end{aligned}
$$

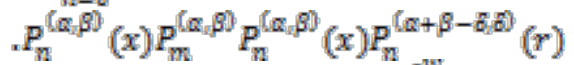

$$
\begin{aligned}
& =a_{n}^{*}(1-r)^{-\sigma}(1+x)^{-\beta} \int_{-1}^{w} \eta(y)(r-y)^{\mu-1}(x-y)^{\beta+\mu-\sigma-1} d y \\
& =a_{n}{ }^{*}(1-r)^{-\delta}(1+x)^{-\beta} \cdot S_{w}(r, x) \\
& \text { where, } w=\min (x, y) \text { and } \eta(y)=(1+y)^{\tilde{d}-\mu}(1-y)^{\tilde{g}-\alpha+\beta}
\end{aligned}
$$

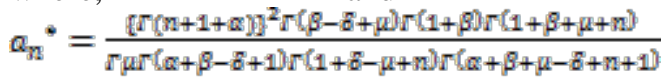

It is assumed that parameters are so constrained that $a_{n}{ }^{*}$ is independent of $\mathrm{n}$, this is of course possible when, for instance $\alpha=v$ $=\lambda-\mu^{\prime}$ and $\beta=\delta$.

\section{THE SOLUTION}

(i) The solution of $\mathrm{N}$-series equations of the first kind involving Jacobi polynomials of different indices :

Let us assume

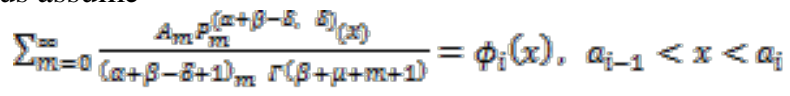

where, $\mathrm{i}=2,4,6, \ldots \ldots, \mathrm{n}$.

and where $\phi_{i}(x)$ are unspecified functions. Using orthogonality relation it follows from equations (1) and (12)

$$
\begin{array}{r}
A_{m}=\frac{(\alpha+\beta-\delta+1)_{m} \Gamma(\beta+\mu+m+1)(2 m+\alpha+\beta+1) \Gamma(m+1) \Gamma(m+\alpha+\beta+1)}{2^{\alpha+\beta+1} \Gamma(m+\alpha+\beta-\delta+1) \Gamma(m+\delta+1)} \\
\sum_{i=0}^{\frac{m-2}{2}}\left\{\int_{a_{2 i}}^{a_{2 i+1}} f_{2 i+1}(x)+\int_{a_{2 i+1}}^{a_{2 i+2}} \phi_{2 i+2}(x)\right\} \quad P_{m}^{[\alpha+\beta-\theta)}(x)(1-x)^{\alpha+\beta-\sigma}(1+x)^{d} d x
\end{array}
$$

Substituting this value of $A_{m}$ in equation (2) and interchanging the order of integration and summation, we get

where

$$
\begin{aligned}
& \sum_{m=0}^{m} \frac{(\alpha+\beta-\delta+1)_{m} \Gamma(\beta+\mu+m+1)(2 m+\alpha+\beta+1) \Gamma(m+1) \Gamma(m+\alpha+\beta+1)}{2^{\alpha+\beta+1} \Gamma(m+\alpha+\beta-\delta+1) \Gamma(m+\delta+1)(\beta+1)_{m} \Gamma(\alpha-\mu+m+1)} \\
& \sum_{\mathrm{i}=0}^{\frac{\mathrm{n}-2}{2}}\left\{\int_{\mathrm{a}_{2 \mathrm{i}}}^{\mathrm{a}_{2 \mathrm{i}+1}} \mathrm{f}_{2 \mathrm{i}+1}(\mathrm{r})+\int_{\mathrm{a}_{2 \mathrm{i}+1}}^{\mathrm{a}_{2 \mathrm{i}+2}} \Phi_{2 \mathrm{i}+2}(\mathrm{r})\right\} . P_{m}^{[\alpha+\beta-\tilde{b})}(x) P_{m}^{[(\alpha, \beta)}(x)(1-r)^{\alpha+\beta-\tilde{b}}(1+r)^{\tilde{d}} d r=f_{j}(x) \\
& a_{j-1}<x<a_{j}, j=2,4,6, \ldots, n \\
& \sum_{i=0}^{\frac{n-z}{2}} \int_{a_{2 i+1}}^{a_{2 i+2}} \emptyset_{2 i+2}(r)(1-r)^{\alpha+\beta-\tilde{\sigma}}(1+r)^{\tilde{\sigma}} . S(r, x) d r=M_{j}(x) \quad a_{j-1}<x<a_{j a} \quad j=2,4,6, \ldots, \ldots, n .
\end{aligned}
$$

$$
M_{j}(x)=f_{j}(x)-\sum_{i=0}^{\frac{n-2}{2}} \int_{a_{2 i}}^{a_{2 i+1}} f_{2 i+1}(r)(1-r)^{\alpha+\beta-\delta}(1+r)^{\tilde{\sigma}} . S(r, x) d r
$$

for all $\mathbf{j}=2,4,6, \ldots \ldots \mathrm{n}$.

Taking $\mathrm{j}=\mathrm{k}$ in equation (14), where $\mathrm{k}$ is an even integer and $2 \leq k \leq n$ and $n$ is the total number of considered equations, we get

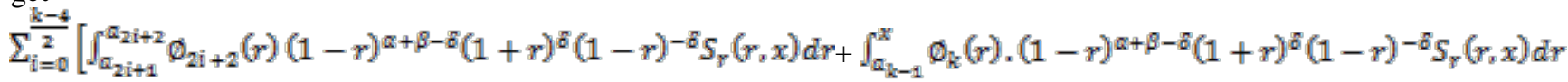

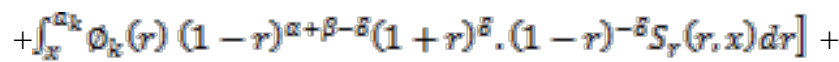

$$
\begin{aligned}
& \sum_{\mathrm{i}=\frac{k}{2}}^{\frac{n-\pi}{2}} \int_{a_{2 i+1}}^{a_{2 i+2}} \emptyset_{2 \mathrm{i}+2}(r)(1-r)^{\alpha+\beta-\sigma}(1+r)^{\tilde{a}}(1-r)^{-\delta} S_{y}(r, x) d r=\frac{(1+x)^{\beta}}{a_{n} *} M_{k}(x)
\end{aligned}
$$


Or

$$
\begin{aligned}
& \int_{a_{k-1}}^{x} \emptyset_{k}(x)(1-r)^{\alpha+\beta-2 \sigma}(1+r)^{\sigma} \int_{0}^{r} \eta(y)(r-y)^{\mu-1}(x-y)^{\beta+\mu-\tilde{\alpha}-1} d y d r \\
& +\int_{x}^{a_{k}} \emptyset_{k}(r)(1-r)^{\alpha+\beta-2 \sigma}(1+r)^{d} \int_{0}^{x} \eta(y)(r-y)^{\mu-1}(x-y)^{\beta+\mu-\delta-1} d y d r \\
& =\frac{(1+x)^{\beta}}{a_{n} *} M_{k}(x)-\sum_{i=0}^{\frac{k i-4}{2}} \int_{a_{2 i+1}}^{a_{2 i+2}} \emptyset_{2 i+2}(r) \cdot(1-r)^{\alpha+\beta-2 \delta}(1+r)^{\tilde{a}} \\
& \int_{0}^{\gamma} \eta(y)(r-y)^{\mu-1}(x-y)^{\beta+\mu-\tilde{d}-1} d y d r-\sum_{i=k / 2}^{\frac{n-2}{2}} \int_{a_{2 i+1}}^{a_{2 i+2}} \emptyset_{2 i+2}(r) \text {. } \\
& (1-r)^{\alpha+\beta-2 \delta}(1+r)^{\tilde{d}} \int_{0}^{x} \eta(y)(r-y)^{\mu-1}(x-y)^{\beta+\mu-\tilde{\theta}-1} d y d r \text {. }
\end{aligned}
$$

Inverting the order of integration,

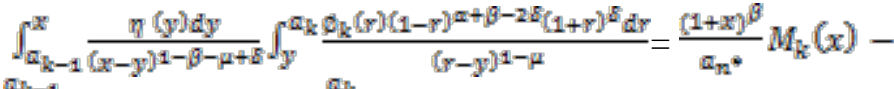

$$
\begin{aligned}
& \int_{0}^{a_{k-1}} \frac{\eta(y) d y}{(x-y)^{1-\beta-\mu+\sigma}} \int_{a_{k-1}}^{a_{k}} \frac{\emptyset_{k}(r)(1-r)^{\alpha+\beta-2 \sigma}(1+r)^{d} d r}{(r-y)^{1-\mu}}
\end{aligned}
$$

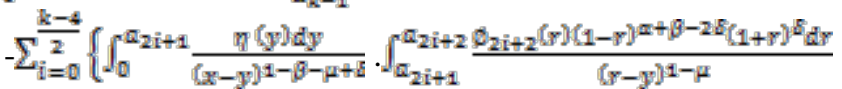

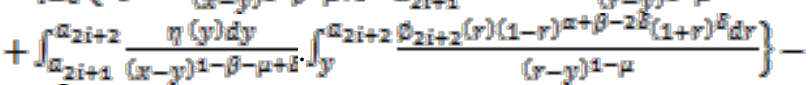

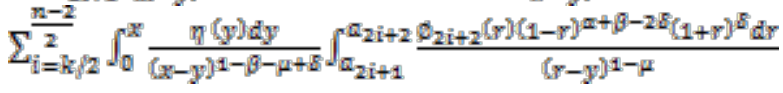

Assuming

For all $\mathrm{k}=2,4,6, \ldots, \mathrm{n}$

$$
\int_{y}^{a_{k} \oint_{k}(r)(1-r)^{\mu+\rho-2} \varepsilon_{(1+r)^{*}} \varepsilon_{d y}}=\bar{\emptyset}_{k}(y), a_{k-1}<x<a_{k}
$$

with the help of the equation (17), the equation (16) takes the form

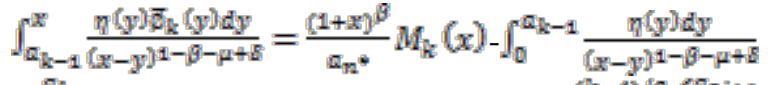

$$
\begin{aligned}
& \cdot \int_{\mathbb{a}_{k-1}}^{a_{k}} \frac{\emptyset_{k}(r)(1-r)^{\alpha+\beta-2 \delta}(1+r)^{\tilde{\sigma}} d r}{(r-y)^{1-\mu}}-\sum_{i=0}^{(k-4) / 2}\left\{\int_{0}^{a_{2 i+1}} \frac{\eta(y) d y}{(x-y)^{1-\beta-\mu+\sigma}}\right. \\
& \left.\cdot \int_{\mathbb{a}_{2 i+1}}^{\mathbb{a}_{k-1}} \frac{\emptyset_{2 i+2}(r)(1-r)^{\alpha+\beta-2 \delta}(1+r)^{\tilde{b}} d r}{(r-y)^{1-\mu}}+\int_{\mathbb{a}_{2 i+1}}^{\mathbb{a}_{2 i+2}} \frac{\Phi_{2 i+2}(y) \eta(y) d y}{(x-y)^{1-\beta-\mu+\delta}}\right\}
\end{aligned}
$$

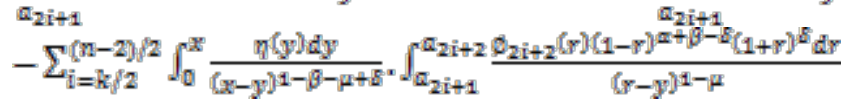

This equation is an Abel type integralequation and its solution is given by

$$
\begin{aligned}
& \eta(\mathrm{t}) \bar{\Phi}_{k}(y)=F_{k}(y)-\frac{\sin (1-\beta-\mu+\delta) \pi}{\pi a_{n} *}\left[\int_{0}^{a_{k-1}} \eta(\xi) d \xi \frac{d}{d y} \int_{a_{k-1}}^{y} \frac{d x}{(y-x)^{\beta+\mu-\delta}(x-\xi)^{1-\beta-\mu+\delta}}\right. \\
& \int_{a_{k-1}}^{a_{k}} \frac{\emptyset_{k}(r)(1-r)^{\alpha+\beta-2 \delta}(1+r)^{\tilde{b}} d r}{(r-\xi)^{1-\mu}}+\sum_{i=0}^{(k-4) / 2} \int_{a_{2 i+2}}^{a_{2 i+1}} \eta(\xi) d \xi \frac{d}{d y} \int_{0}^{y} \frac{d x}{(y-x)^{\beta+\mu-\sigma}(x-\xi)^{1-\beta-\mu+\delta}} \\
& \left.\int_{\mathbb{a}_{2 i+1}}^{a_{\mathbb{a}_{2 i+2}-1}} \frac{\emptyset_{2 i+2}(r)(1-r)^{\alpha+\beta-2 \delta}(1+r)^{\tilde{b}} d r}{(r-\xi)^{1-\mu}}+\int_{\mathbb{a}_{2 i+1}}^{\mathbb{a}_{2 i+2}} \eta(\xi) \bar{\emptyset}_{2 i+2}(\xi) d \xi \times \frac{d}{d y} \int_{a_{k-1}}^{y} \frac{d x}{(y-x)^{\beta+\mu-\delta}(x-\xi)^{1-\beta-\mu+\varepsilon}}\right\}
\end{aligned}
$$

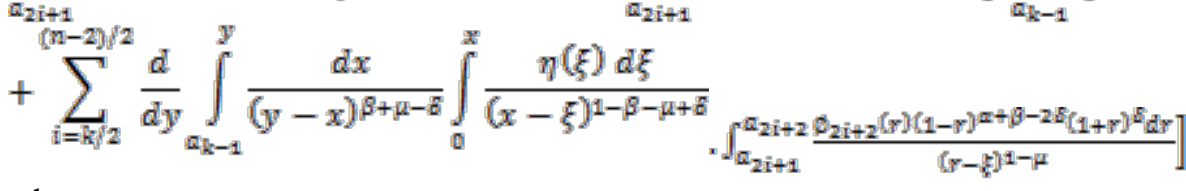

where 


$$
F_{k}(y)=\frac{\sin (1-\beta-\mu+\delta) \pi}{\pi a_{n}^{3}} \frac{d}{d y} \int_{a_{k-1}}^{y} \frac{(1+x)^{g} M_{k}(x) d x}{(y-x)^{\beta+\mu-\varepsilon}}
$$

Changing the order of integration of the last integral of equation (26), we get

$$
\begin{aligned}
& \eta(y) \bar{\Phi}_{k}(y)=F_{k}(y)-\frac{\sin (1-\beta-\mu+\delta) \pi}{\pi}\left[\int_{0}^{\alpha_{k-1}} \eta(\xi) d \xi \frac{d}{d y} \int_{a_{k-1}}^{y} \frac{d x}{(y-x)^{\beta+\mu-\delta}(x-\xi)^{1-\beta-\mu+\delta}}\right. \\
& \int_{a_{k-1}}^{a_{k}} \frac{\emptyset_{k}(r)(1-r)^{\alpha+\beta-2 \sigma}(1+r)^{\delta} d r}{(r-\xi)^{1-\mu}} \\
& +\sum_{i=0}^{(k-4) / 2}\left\{\int_{0}^{a_{2 i+1}} \eta(\xi) d \xi \frac{d}{d y} \cdot \int_{a_{k-1}}^{y} \frac{d x}{(y-x)^{\beta+\mu-\delta}(x-\xi)^{1-\beta-\mu+b}} \int_{a_{2 i+1}}^{a_{2 i+2}} \frac{\emptyset_{2 i+2}(r)(1-r)^{\alpha+\beta-2 \delta}(1+r)^{d} d r}{(r-\xi)^{1-\mu}}\right.
\end{aligned}
$$

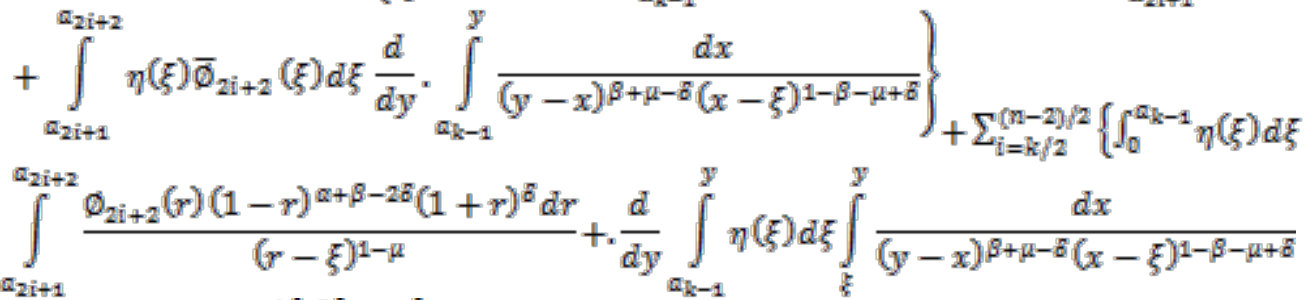

$$
\begin{aligned}
& \left.\left.\cdot \int_{a_{2 i+1}}^{a_{2 i+2}} \frac{\phi_{2 i+2}(r)(1-r)^{\left.\mu+\beta-2 \varepsilon_{(1+r}\right)^{\delta}} d r}{(r-\xi)^{1-\mu}}\right\}\right]
\end{aligned}
$$

Using these equations,

$$
\begin{aligned}
& \int_{\xi}^{y} \frac{d x}{(y-x)^{\beta+\mu-Q(x-\xi)^{1-(\beta-\mu+8}}=\frac{\pi}{\sin (1-\beta-\mu+\delta) \pi}} \\
& \text { And } \frac{d}{d y} \int_{\alpha}^{y} \frac{d x}{(y-x)^{1-m}(x-t)^{m}}=\frac{(\alpha-t)^{1-m}}{(y-t)(y-a)^{1-m}}
\end{aligned}
$$

Equation (21) will be ,

$$
\begin{aligned}
& \eta(y) \bar{\emptyset}_{k}(y)=F_{k}(y)-\frac{\sin (1-\beta-\mu+\delta) \pi}{\pi}\left[\int_{0}^{a_{k-1}} \frac{\eta(\xi)\left(a_{k-1}-\xi\right)^{\beta+\mu-\delta} d \xi}{\left(y-a_{k-1}\right)^{\beta+\mu-\delta}(y-\xi)}\right. \\
& =\int_{a_{k-1}}^{a_{k}} \frac{\emptyset_{k}(r)(1-r)^{\alpha+\beta-2 b}(1+r)^{d} d r}{(r-\xi)^{1-\mu}}+\sum_{i=0}^{(k-4) / 2}\left\{\int_{0}^{a_{2 i+1}} \frac{\eta(\xi)\left(a_{k-1}-\xi\right)^{\beta+\mu-\sigma} d \xi}{\left(y-a_{k-1}\right)^{\beta+\mu-\sigma}(y-\xi)}\right. \\
& \left.\cdot \int_{a_{2 i+1}}^{a_{k-1}} \frac{\emptyset_{2 i+2}(r)(1-r)^{\alpha+\beta-2 b}(1+r)^{a} d r}{(r-\xi)^{1-\mu}} \int_{a_{2 i+1}}^{a_{2 i+2}} \frac{\left.\eta(\xi)\left(a_{k-1}-\xi\right)^{\beta+\mu-\sigma} \bar{\phi}_{2 i+2}(\xi) d \xi\right)}{\left(y-a_{k-1}\right)^{\beta+\mu-\sigma}(y-\xi)}\right\} \\
& +\sum_{i=k / 2}^{a_{2 i+1}}\left[\int_{0}^{(n-2) / 2} \frac{\eta(\xi)\left(a_{k-1}-\xi\right)^{\beta+\mu-\sigma} d \xi}{\left(y-a_{k-1}\right)^{\beta+\mu-\delta}(y-\xi)} \int_{a_{2 i+1}}^{a_{k-1}} \frac{\phi_{2 i+2}(r)(1-r)^{\alpha+\beta-2 \delta}(1+r)^{\sigma} d r}{(r-\xi)^{1-\mu}}\right] \\
& -\sum_{i=k / 2}^{(n-2) / 2} \frac{d}{d y} \int_{a_{k-1}}^{y} \eta(\xi) d \xi \int_{a_{2 i+1}}^{a_{2 i+2}} \frac{\phi_{2 i+2}(r)(1-r)^{\mu+19-2 \varepsilon_{(1+r)} \varepsilon d r}}{(r-\xi)^{1-\mu}}
\end{aligned}
$$

Equation (24) is also Abel type integral equation. Therefore its solution is given by

for all $\mathrm{k}=2,4,6, \ldots \ldots \mathrm{n}$.

$$
\emptyset_{k}(r)(1-r)^{\alpha+\beta-2 \sigma}(1+r)^{a}=\frac{-\sin (1-\mu) \pi}{\pi} \frac{d}{d y} \int_{r}^{a_{k}} \frac{\bar{\phi}_{k}(y) d y}{\mathbb{4}(y-r)^{\mu}}
$$

Therefore,

$$
\int_{a_{k-1}}^{a_{k}} \frac{\phi_{k}(r)(1-r)^{\left.\alpha+\beta-2 \varepsilon_{(1+r}\right)^{8} d y}}{(y-\xi)^{1-\mu}}=\frac{\sin (1-\mu) \pi}{\pi\left(a_{k-1}-\xi\right)^{-\mu \mu^{\mu}}} \int_{a_{k-1}}^{a_{k}} \frac{\Phi_{k}(y) d y}{\left(y-b\left(y-a_{k-1}\right)^{\mu}\right.}
$$

for all $\mathrm{k}=2,4,6, \ldots \ldots \mathrm{n}$.

Applying the above result in equation (24) and also applying the Leibnitz theorem we get

$$
\eta(y) \bar{\Phi}_{k}(y)=F_{k}(y)-\frac{\sin (1-\beta-\mu+\delta) \pi \sin (1-\mu) \pi}{\pi^{2}\left(y-a_{k-1}\right)^{\beta+\mu-\sigma}}\left[\int_{0}^{a_{k-1}} \frac{\eta(\xi)\left(a_{k-1}-\xi\right)^{\beta+\mu-\varepsilon_{j}} d \xi}{(y-\xi)\left(a_{k-1}-\xi\right)^{-\mu}} \int_{a_{k-1}}^{a_{k}} \frac{\bar{\emptyset}_{k}(t) d t}{\left(t-a_{k-1}\right)^{\mu}(t-\xi)}\right.
$$




$$
\begin{aligned}
& \sum_{i=0}^{(k-4) / 2}\left\{\int_{0}^{a_{2 i+1}} \frac{\eta(\xi)\left(a_{k-1}-\xi\right)^{\beta+\mu-\delta} d \xi}{(y-\xi)\left(a_{k-1}-\xi\right)^{-\mu}} \cdot \int_{a_{2 i+1}}^{a_{2 i+2}} \frac{\bar{\emptyset}_{2 i+2}(t) d t}{(t-\xi)\left(t-a_{2 i+1}\right)^{\mu}}\right. \\
& \left.-\mu \int_{a_{2 i+1}}^{a_{2 i+2}} \bar{\emptyset}_{2 i+2}(t) d t \int_{a_{2 i+1}}^{t} \frac{d r}{(t-r)^{1+\mu}} \cdot \int_{a_{2 i+1}}^{r} \frac{\eta(\xi)\left(a_{k-1}-\xi\right)^{\beta+\mu-\delta^{-}} d \xi}{(y-\xi)(r-\xi)^{1-\mu}}\right\} \\
& \left.+\sum_{i=k / 2}^{\frac{a_{2 i}}{2}} \int_{0}^{a_{k-1}} \frac{\eta(\xi)\left(a_{k-1}-\xi\right)^{\beta+\mu-\varepsilon_{j}} d \xi}{(y-\xi)\left(a_{k-1}-\xi\right)^{-\mu}} \cdot \int_{a_{2 i+1}}^{a_{2 i+2}} \frac{\bar{\emptyset}_{2 i+2}(t) d t}{(t-\xi)\left(t-a_{2 i+1}\right)^{\mu}}\right] \\
& -\sum_{i=k / 2}^{(n-2) / 2} \frac{\sin (1-\mu) \pi}{\pi} \cdot \frac{d}{d y} \int_{a_{k-1}}^{y} \eta(\xi)\left(a_{2 i+1}-\xi\right)^{\mu} d \xi \\
& \int_{a_{2 i+1}}^{a_{2 i+2}} \frac{\Phi_{2 i+2}(t) d t}{(t-b)\left(t-a_{2 i+1}\right)^{\mu}}
\end{aligned}
$$

This equation can be written as,

$$
\begin{aligned}
\eta(y) \bar{\Phi}_{k}(y) & =F_{k}(y)-\int_{a_{k-1}}^{a_{k}} \bar{\emptyset}_{k}(t) L_{k}(\mathrm{t}, \mathrm{y}) \mathrm{dt} \\
& -\sum_{i=0}^{(k-4) / 2} \int_{a_{2 i+1}}^{a_{2 i+2}} \bar{\Phi}_{2 i+2}(t) R_{2 i+2}(t, y) d t \\
& -\sum_{i=k / 2}^{(n-2) / 2} \int_{a_{2 i+1}}^{a_{2 i+2}} \bar{\emptyset}_{2 i+2}(t) T_{2 i+2}(t, y) d t \quad a_{k-1}<r<a_{k}
\end{aligned}
$$

where,

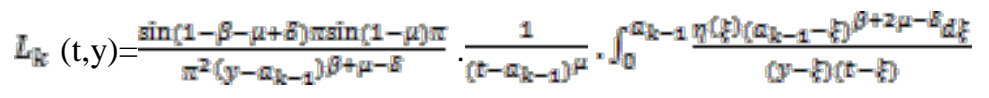

$R_{2 i+2}\left(t_{v} y\right)=\frac{\sin (1-\beta-\mu+\delta) \pi \sin (1-\mu) \pi}{\pi^{2}\left(y-a_{k-1}\right)^{\beta+\mu-\delta}}\left\{\frac{1}{\left(t-a_{2 i-1}\right)^{\mu}} \cdot \int_{0}^{a_{2 i+1}} \frac{\eta(\xi)\left(a_{k-1}-\xi\right)^{\beta+2 \mu-\delta}\left(a_{2 i+1}-\xi\right)^{\mu} d \xi}{(y-\xi)(t-\xi)}\right.$

$$
\left.\int_{a_{2 i+1}}^{\gamma} \frac{\left.\eta(\xi)\left(a_{k-1}-k\right)^{9+\mu-U_{d k}}\right\}}{(y-b)\left(r-k^{k}\right)^{1-\mu}}\right\}
$$

$$
\begin{aligned}
T_{2 i+2}(t, y) & =\frac{\sin (1-\mu) \pi}{\pi} \frac{1}{\left(t-a_{2 i}+1\right)^{\mu}}\left\{\frac{\sin (1-\beta-\mu+\delta) \pi}{\pi\left(y-a_{k-1}\right)^{\beta+\mu-\delta}} \int_{0}^{a_{k-1}} \frac{\eta(\xi)\left(a_{k-1}-\xi\right)^{\beta+\mu-\tilde{x}}\left(a_{2 i+1}-\xi\right)^{\mu} d \xi}{(y-\xi)(t-\xi)}\right. \\
& \left.+\frac{d}{d y} \cdot \int_{a_{k-1}}^{y} \frac{\eta(\xi)\left(a_{2 i+1}-\hat{b}\right)+\xi}{(t-b)}\right\}
\end{aligned}
$$

Substituting $\mathrm{k}=2,4,6, \ldots \ldots, \mathrm{n}_{\mathrm{r}}$ in equation (28) we will get $\mathrm{n} / 2$ simultaneous Fredholm Integral equations of the second kind with the help of these $n / 2$ simultaneous equations we can calculate $\bar{\emptyset}_{2}(y), \bar{\emptyset}_{4}(y), \ldots \ldots \bar{\Phi}_{n}(y)$ and Then the values of $\emptyset_{2}(x), \emptyset_{4}(x), \ldots . \emptyset_{n}(x)$ can be determined. After all these calculations we can compute the coefficient $A_{m}$ with the help of equation (13).

(ii) The solution of N-series equations of the second kind involving Jacobi polynomials of different indices :

Let us assume

$$
\sum_{m=0}^{\infty} \frac{D_{m} P_{m}^{(\alpha, \beta)}(x)}{(\beta+1)_{m}[(\alpha-\mu+m+1)}=\Psi_{i}(x), \quad a_{i-1}<x<a_{i}
$$

where, $\mathrm{i}=1,3,5, \ldots \ldots \mathrm{n}-1$. and where $\Psi_{i}(x)$ are unspecified functions. Using orthogonality relation it follows from equations (1) and (32)

$$
\begin{aligned}
D_{m}= & \frac{(\alpha+\beta-\delta+1)_{m} \Gamma(\beta+\mu+m+1)(2 m+\alpha+\beta+1) \Gamma(m+1) \Gamma(m+\alpha+\beta+1)}{2^{\alpha+\beta+1} \Gamma(m+\alpha+\beta-\delta+1) \Gamma(m+\delta+1)} \\
& \sum_{i=0}^{\frac{n-2}{2}}\left\{\int_{a_{2 i+1}}^{a_{2 i+2}} f_{2 i+2}(x)+\int_{a_{2 i}}^{a_{2 i+1}} \varphi_{2 i+1}(x)\right\} \quad P_{m}^{(\alpha+\beta-b)}(x)(1-x)^{\alpha+\beta-\sigma_{0}}(1+x)^{\delta} d x .
\end{aligned}
$$

Substituting this value of $D_{m}$ in equation (5) and interchanging the order of integration and summation, we get

$$
\sum_{m=0}^{m} \frac{(\alpha+\beta-\delta+1)_{m} \Gamma(\beta+\mu+m+1)(2 m+\alpha+\beta+1) \Gamma(m+1) \Gamma(m+\alpha+\beta+1)}{2^{\alpha+\beta+1} \Gamma(m+\alpha+\beta-\delta+1) \Gamma(m+\delta+1)(\beta+1)_{m} \Gamma(\alpha-\mu+m+1)}
$$




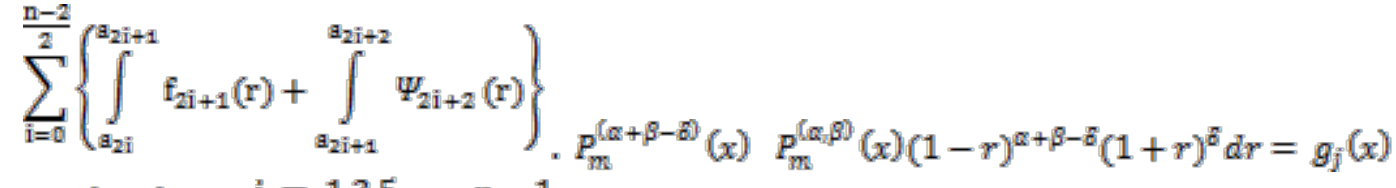

$$
\begin{aligned}
& a_{j-1}<x<a_{j}, \mathfrak{j}=1,3,5, \ldots \ldots, \mathrm{n}-1 \\
& \sum_{i=0}^{\frac{n-2}{2}} \int_{a_{2 i}}^{a_{2 i+1}} \Psi_{2 i+1}(r)(1-r)^{\alpha+\beta-\sigma}(1+r)^{\tilde{a}} \cdot S(r, x) d r=N_{j}(x) \\
& a_{j-1}<x<a_{j_{a}} \quad \mathrm{j}=1,3,5, \ldots, \mathrm{n}-1 .
\end{aligned}
$$

where

for all $\mathfrak{j}=1,3,5, \ldots, n-1$.

$$
N_{j}(x)=g_{j}(x)-\sum_{i=0}^{\frac{n-2}{2}} \int_{a_{2 i+1}}^{a_{2 i+2}} f_{2 i+2}(r)(1-r)^{\alpha+\beta-\tilde{\delta}}(1+r)^{\tilde{\delta}} S(r, x) d r(36)
$$

Taking $\mathbf{j}=\mathrm{k}$ in equation (34), where $\mathrm{k}$ is an odd integer and $1 \leq k \leq n-1$ and $n$ is the total number of considered equations, we get

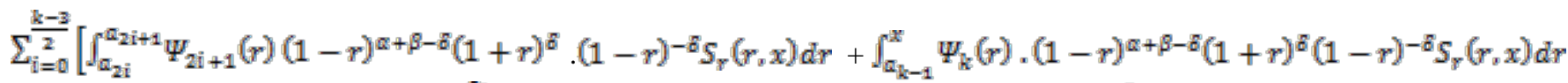

$$
\begin{aligned}
& \left.+\int_{x}^{a_{k}} \Psi_{k}(r)(1-r)^{\alpha+\beta-\tilde{d}}(1+r)^{\tilde{d}} \cdot(1-r)^{-{ }^{\tilde{b}}} S_{y}(r, x) d r\right]+ \\
& \sum_{\mathrm{i}=\frac{k+1}{2}}^{\frac{n-z}{2}} \int_{a_{2 i}}^{a_{2 i+1}} \Psi_{2 i+1}(r)(1-r)^{\alpha+\beta-\tilde{\varepsilon}}(1+r)^{\tilde{\sigma}} \cdot(1-r)^{-\tilde{\varepsilon}} S_{y}(r, x) d r=\frac{(1+x)^{\beta}}{a_{n} *} N_{k}(x) \\
& \int_{a_{k-1}}^{x} \Psi_{k}(x)(1-r)^{\alpha+\beta-2 \delta}(1+r)^{\sigma} \int_{0}^{r} \eta(y)(r-y)^{\mu-1}(x-y)^{\beta+\mu-\sigma-1} d y d r \\
& +\int_{x}^{a_{k}} \Psi_{k}(r)(1-r)^{\alpha+\beta-2 \delta}(1+r)^{a} \int_{0}^{x} \eta(y)(r-y)^{\mu-1}(x-y)^{\beta+\mu-\varepsilon-1} d y d r \\
& =\frac{(1+x)^{\beta}}{a_{n} *} N_{k}(x)-\sum_{i=0}^{\frac{k-a}{2}} \int_{a_{2 i}}^{a_{2 i+1}} \Psi_{2 i+1}(r) \cdot(1-r)^{\alpha+\beta-2 \tilde{b}}(1+r)^{\tilde{d}} \\
& \int_{0}^{r} \eta(y)(r-y)^{\mu-1}(x-y)^{\beta+\mu-\delta-1} d y d r- \\
& \sum_{i=\{k+1) / 2}^{\frac{n-2}{2}} \int_{a_{2 i}}^{a_{2 i+1}} \Psi_{2 i+1}(r) \cdot(1-r)^{\alpha+\beta-2 \delta}(1+r)^{\tilde{\sigma}} \\
& \int_{0}^{x} \eta(y)(r-y)^{\mu-1}(x-y)^{\beta+\mu-\tilde{b}-1} d y d r .
\end{aligned}
$$

Inverting the order of integration,

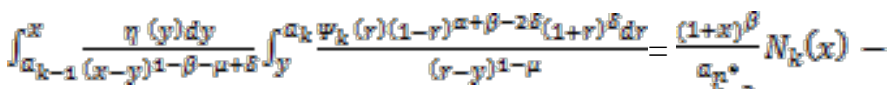

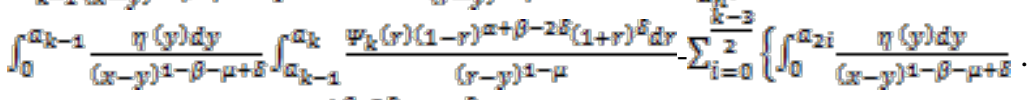

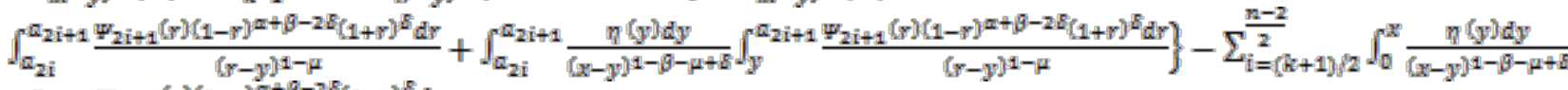

$$
\begin{aligned}
& \int_{a_{2 i}}^{a_{2 i+1}} \frac{\Psi_{2 i+1}(r)(1-r)^{\pi+\beta-2 \varepsilon_{(1+r)} v d r}}{(r-y)^{1-\mu}}
\end{aligned}
$$

Assuming

$$
\int_{y}^{a_{k}} \frac{\Psi_{k}(r)(1-r)^{a+\beta-2} \varepsilon_{(1+r)^{s}} d y}{(r-y)^{1-\mu}}=\overline{\Psi_{k}}(y), a_{k-1}<x<a_{k}
$$

For all $\mathrm{k}=1,3,5, \ldots \ldots, \mathrm{n}-1$.

With the help of the equation (38), the equation (37) takes the form, 


$$
\begin{aligned}
& \int_{\mathbb{a}_{k-1}}^{x} \frac{\eta(y) \bar{\Psi}_{k}(y) d y}{(x-y)^{1-\beta-\mu+\sigma}}=\frac{(1+x)^{\beta}}{a_{n} *} N_{k}(x)-\int_{0}^{a_{k-1}} \frac{\eta(y) d y}{(x-y)^{1-\beta-\mu+\sigma}} \\
& \cdot \int_{\mathbb{a}_{k-1}}^{a_{k}} \frac{\Psi_{k}(r)(1-r)^{\alpha+\beta-2 B}(1+r)^{\tilde{d}} d r}{(r-y)^{1-\mu}}-\sum_{i=1}^{(k-1) / 2}\left\{\int_{0}^{a_{2 i}} \frac{\eta(y) d y}{(x-y)^{1-\beta-\mu+\delta}}\right. \\
& \left.\cdot \int_{a_{2 i}}^{a_{2 i+1}} \frac{\Psi_{2 i+1}(r)(1-r)^{\alpha+\beta-2 \delta}(1+r)^{\delta} d r}{(r-y)^{1-\mu}}+\int_{a_{2 i}}^{a_{2 i+1}} \frac{\bar{\Psi}_{2 i+1}(y) \eta(y) d y}{(x-y)^{1-\beta-\mu+\delta}}\right\}
\end{aligned}
$$

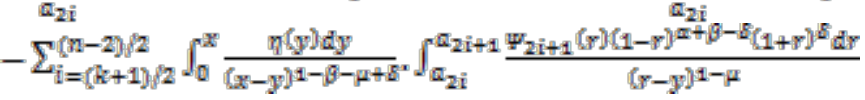

This equation is an Abel type integral equation and its solution is given by

$$
\begin{aligned}
& \eta(\mathrm{t}) \bar{\Psi}_{k}(y)=G_{k}(y)-\frac{\sin (1-\beta-\mu+\delta) \pi}{\pi a_{n} *}\left[\int_{0}^{a_{k}-1} \eta(\xi) d \xi \frac{d}{d y}\right. \\
& \int_{a_{k-1}}^{y} \frac{d x}{(y-x)^{\beta+\mu-\varepsilon}(x-\xi)^{1-\beta-\mu+\delta}} \int_{a_{k-1}}^{a_{k}} \frac{\Psi_{k}(r)(1-r)^{\alpha+\beta-2 \varepsilon}(1+r)^{z} d r}{(r-\xi)^{1-\mu}}+ \\
& \sum_{i=1}^{(k-2) / 2}\left\{\int_{0}^{a_{2 i}} \eta(\xi) d \xi \frac{d}{d y} \int_{a_{k-1}}^{y} \frac{d x}{(y-x)^{\beta+\mu-\varepsilon}(x-\xi)^{1-\beta-\mu+\sigma}}\right. \\
& \int_{a_{2 i}}^{a_{2 i+1}} \frac{\Psi_{2 i+1}(r)(1-r)^{\alpha+\beta-2 \delta}(1+r)^{\tilde{b}} d r}{(r-\xi)^{1-\mu}}+\int_{\mathbb{a}_{2 i}}^{a_{2 i+1}} \eta(\xi) \bar{\Psi}_{2 i+1}(\xi) d \xi \\
& \left.\cdot \frac{d}{d y} \int_{\mathbb{a}_{k-1}}^{y} \frac{d x}{(y-x)^{\beta+\mu-\delta}(x-\xi)^{1-\beta-\mu+\varepsilon}}\right\}+\sum_{i=\{(k+1) / 2}^{\substack{\mathbb{a}_{2 i} \\
(n-2) / 2}} \frac{d}{d y} \int_{\mathbb{a}_{k-1}}^{y} \frac{d x}{(y-x)^{\beta+\mu-\delta}}
\end{aligned}
$$

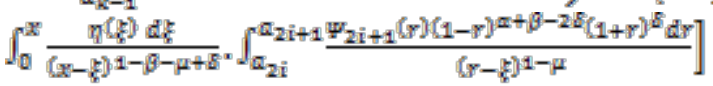

where

$$
G_{k}(y)=\frac{\sin (1-\beta-\mu+\tilde{b}) \pi}{\pi a_{n}^{*}} \frac{d}{d y} \int_{a_{k-1}}^{y} \frac{(1+x)^{\beta^{\beta_{k}}}(x) d x}{(y-x)^{9+\mu-\varepsilon}}
$$

Changing the order of integration of the last integral of equation (40), we get

$$
\eta(y) \bar{\Psi}_{k}(y)=G_{k}(y)-\frac{\sin (1-\beta-\mu+\delta) \pi}{\pi}\left[\int_{0}^{a_{k-1}} \eta(\xi) d \xi \frac{d}{d y}\right.
$$

$$
\begin{aligned}
& \int_{a_{k-1}}^{y} \frac{d x}{(y-x)^{\beta+\mu-\sigma}(x-\xi)^{1-\beta-\mu+\sigma}} \cdot \int_{a_{k-1}}^{a_{k}} \frac{\Psi_{k}(r)(1-r)^{\alpha+\beta-2 \sigma}(1+r)^{b} d r}{(r-\xi)^{1-\mu}} \\
& +\sum_{i=1}^{(k-2) / 2}\left\{\int_{0}^{a_{2 i}} \eta(\xi) d \xi \frac{d}{d y} \int_{a_{k-1}}^{y} \frac{d x}{(y-x)^{\beta+\mu-\delta}(x-\xi)^{1-\beta-\mu+\delta}}\right. \\
& \int_{\mathbb{a}_{2 i}}^{a_{2 i+1}} \frac{\Psi_{2 i+1}(r)(1-r)^{\alpha+\beta-2 \delta}(1+r)^{\tilde{d}} d r}{(r-\xi)^{1-\mu}}+\int_{a_{2 i}}^{{a_{2 i}}_{1+1}} \eta(\xi) \bar{\Psi}_{2 i+1}(\xi) d \xi
\end{aligned}
$$

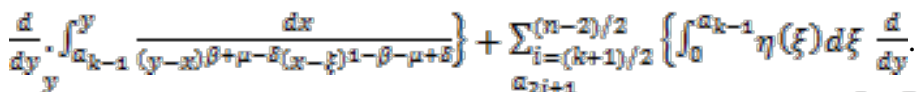

$$
\begin{aligned}
& \int_{a_{k-1}}^{y} \frac{d x}{(y-x)^{\beta+\mu-\delta}(x-\xi)^{1-\beta-\mu+\delta}} \cdot \int_{\alpha_{2 i}}^{a_{2 i+1}} \frac{\Psi_{2 i+1}(r)(1-r)^{\alpha+\beta-2 \delta}(1+r)^{d} d r}{(r-\xi)^{1-\mu}}+
\end{aligned}
$$




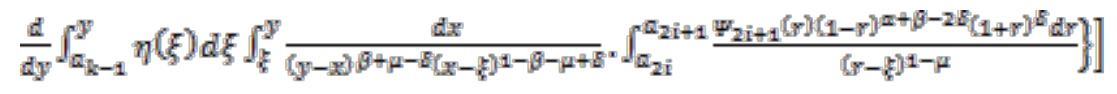

Using these equations,

And

$$
\int_{\xi}^{y} \frac{d x}{(y-x), 9+\mu-\sqrt{2}(x-\xi)^{1-\beta-\mu+\beta+\delta}}=\frac{\pi}{\sin (1-\beta-\mu+\delta) \pi}
$$

Equation (42) will be ,

$$
\begin{aligned}
& \eta(y) \bar{\Psi}_{k}(y)=G_{k}(y)-\frac{\sin (1-\beta-\mu+\delta) \pi}{\pi}\left[\int_{0}^{a_{k-1}} \frac{\eta(\xi)\left(a_{k-1}-\xi\right)^{\beta+\mu-\delta} d \xi}{\left(y-a_{k-1}\right)^{\beta+\mu-\delta}(y-\xi)}\right. \\
& \cdot \int_{a_{k-1}}^{a_{k}} \frac{\Psi_{k}(r)(1-r)^{\alpha+\beta-2 b}(1+r)^{\tilde{b}} d r}{(r-\xi)^{1-\mu}}+\sum_{i=1}^{(k-a) / 2}\left\{\int_{0}^{a_{2 i}} \frac{\eta(\xi)\left(a_{k-1}-\xi\right)^{\beta+\mu-\sigma} d \xi}{\left(y-a_{k-1}\right)^{\beta+\mu-\tilde{b}}(y-\xi)}\right. \\
& \left.\int_{a_{2 i}}^{a_{k i+1}} \frac{\Psi_{2 i+1}(r)(1-r)^{\alpha+\beta-2 \delta}(1+r)^{z} d r}{(r-\xi)^{1-\mu}} \int_{a_{2 i}}^{\substack{a_{2 i+1}}} \frac{\eta(\xi)\left(a_{k-1}-\xi\right)^{\beta+\mu-\delta} \bar{\Psi}_{2 i+1}(\xi) d \xi}{\left(y-a_{k-1}\right)^{\beta+\mu-\delta}(y-\xi)}\right\} \\
& +\sum_{i=[(k+1) / 2}^{a_{2 i}}\left[\int_{0}^{(n-2) / 2} \frac{\eta(\xi)\left(a_{k-1}-\xi\right)^{\beta+\mu-\delta} d \xi}{\left(y-a_{k-1}\right)^{\beta+\mu-\sigma}(y-\xi)} \cdot \int_{a_{2 i}}^{a_{k i}} \frac{\Psi_{2 i+1}(r)(1-r)^{\alpha+\beta-2 \delta}(1+r)^{\delta} d r}{(r-\xi)^{1-\mu}}\right] \\
& -\sum_{i=(k+1) / 2}^{(n-2) / 2} \frac{d}{d y} \int_{a_{k-1}}^{y} \eta(\xi) d \xi \int_{a_{2 i}}^{a_{2 i+1}} \frac{\Psi_{2 i+1}(r)(1-r) \pi+\beta-2 \varepsilon_{(1+r)} \varepsilon^{\delta} d r}{(r-\xi)^{1-\mu}}
\end{aligned}
$$

Equation (45) is also Abel type integral equation. Therefore its solution is given by

$$
\Psi_{k}(r)(1-r)^{\alpha+\beta-2 \tilde{b}}(1+r)^{\tilde{a}}=\frac{-\sin (1-\mu) \pi}{\pi} \frac{d}{d y} \int_{y}^{a_{k} \Phi_{k}(y) d y} \frac{\Psi^{\prime}}{(y-r) \mu}
$$

for all $\mathrm{k}=1,3,5, \ldots . . \mathrm{n}-1$.

Therefore,

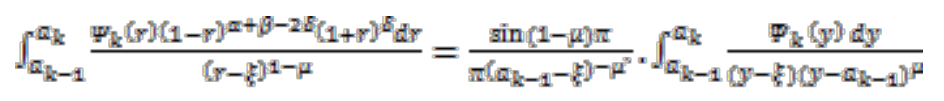

for all $\mathrm{k}=1,3,5, \ldots, \mathrm{n}-1$.

Applying the above result in equation (45) and also applying the Leibnitz theorem, we get

$$
\begin{aligned}
& \eta(y) \bar{\Psi}_{k}(y)=G_{k}(y)-\frac{\sin (1-\beta-\mu+\delta) \pi \sin (1-\mu) \pi}{\pi^{2}\left(y-a_{k-1}\right)^{\beta+\mu-\delta}}\left[\int_{0}^{a_{k-1}} \frac{\eta(\xi)\left(a_{k-1}-\xi\right)^{\beta+\mu-\delta} d \xi}{(y-\xi)\left(a_{k-1}-\xi\right)^{-\mu}}\right. \\
& \int_{a_{k-1}}^{a_{k}} \frac{\bar{\Psi}_{k}(t) d t}{\left(t-a_{k-1}\right)^{\mu}(t-\xi)} \sum_{i=1}^{(k-a) / 2}\left\{\int_{0}^{a_{2 i}} \frac{\eta(\xi)\left(a_{k-1}-\xi\right)^{\beta+\mu-\sigma_{0}} d \xi}{(y-\xi)\left(a_{2 i}-\xi\right)^{-\mu}} \int_{a_{2 i}}^{a_{2 i+1}} \frac{\bar{\Psi}_{2 i+1}(t) d t}{(t-\xi)\left(t-a_{2 i}\right)^{\mu}}\right. \\
& \left.-\mu \int_{a_{2 i}}^{a_{2 i+1}} \bar{\Psi}_{2 i+1}(t) d t \int_{a_{2 i}}^{t} \frac{d r}{(t-r)^{1+\mu}} \int_{a_{2 i}}^{r} \frac{\eta(\xi)\left(a_{k-1}-\xi\right)^{\beta+\mu-\delta} d \xi}{(y-\xi)(r-\xi)^{1-\mu}}\right\} \\
& \left.+\sum_{i=\{(k+1) / 2}^{\frac{a_{2 i}}{2}} \int_{0}^{a_{k-1}} \frac{\eta(\xi)\left(a_{k-1}-\xi\right)^{\beta+\mu-\delta_{i}} d \xi}{(y-\xi)\left(a_{2 i}-\xi\right)^{-\mu}} \int_{a_{2 i}}^{a_{2 i+1}} \frac{\bar{\Psi}_{2 i+1}(t) d t}{(t-\xi)\left(t-a_{2 i}\right)^{\mu}}\right]- \\
& \sum_{i=(k+1) / 2}^{(n-2) / 2} \frac{\sin (1-\mu) \pi}{\pi} \cdot \frac{d}{d y} \int_{a_{k-1}}^{y} \eta(\xi)\left(a_{2 i}-\xi\right)^{\mu} \int_{a_{2 i}}^{a_{2 i+1}} \frac{\Phi_{2 i+1}(t) d t}{(t-k)\left(t-a_{2 i}\right)^{\mu}}
\end{aligned}
$$


This equation can be written as,

$$
\begin{aligned}
& \eta(y) \bar{\Psi}_{k}(y)=G_{k}(y)-\int_{a_{k-1}}^{a_{k}} \bar{\Psi}_{k}(t) P_{k}(\mathrm{t}, \mathrm{y}) \mathrm{dt} \\
& -\sum_{i=1}^{(k-a) / 2} \int_{a_{2 i}}^{a_{2 i+1}} \bar{\Psi}_{2 i+1}(t) Q_{2 i+1}(t, y) d t-\sum_{i=(k+1) / 2} \int_{a_{2 i}}^{(n-2) / 2} \bar{\Psi}_{2 i+1}(t) S_{2 i+1}(t, y) d t
\end{aligned}
$$

Where,

$$
\begin{aligned}
& P_{k}(\mathrm{t}, \mathrm{y})=\frac{\sin (1-\beta-\mu+\sigma) \pi \sin (1-\mu) \pi}{\pi^{2}\left(y-a_{k-1}\right)^{\beta+\mu-\varepsilon}} \frac{1}{\left(\mathrm{t}-a_{\mathrm{k}-1}\right)^{\mu}} \int_{0}^{a_{k-1}} \frac{\eta(k)\left(a_{\mathrm{k}-1}-b^{\beta+2 \mu-\varepsilon_{d k}}\right.}{(y-b)(t-k)} \\
& Q_{2 i+1}\left(t_{i} y\right)=\frac{\sin (1-\beta-\mu+\delta) \pi \sin (1-\mu) \pi}{\pi^{2}\left(y-a_{k-1}\right)^{\beta+\mu-\sigma}}\left\{\frac{1}{\left(t-a_{2 i-1}\right)^{\mu}} \int_{0}^{a_{2 i}} \frac{\eta(\xi)\left(a_{k-1}-\xi\right)^{\beta+2 \mu-\delta}\left(a_{2 i}-\xi\right)^{\mu} d \xi}{(y-\xi)(t-\xi)}\right. \\
& \left.-\mu \int_{a_{2 i}}^{t} \frac{d r}{(t-r)^{1+\mu}} \int_{a_{2 i}}^{y} \frac{\eta(\xi)\left(a_{k-1}-b^{j+\mu-\varepsilon_{d \xi}}\right)}{(y-\xi)(r-\xi)^{1-\mu}}\right) \\
& S_{2 i+1}(t, y)=\frac{\sin (1-\mu) \pi}{\pi} \frac{1}{\left(t-a_{2 i}\right)^{\mu}}\left\{\frac{\sin (1-\beta-\mu+\delta) \pi}{\pi\left(y-a_{k-1}\right)^{\beta+\mu-\delta}} \int_{0}^{a_{k-1}} \frac{\eta(\xi)\left(a_{k-1}-\xi\right)^{\beta+\mu-\sigma}\left(a_{2 i}-\xi\right)^{\mu} d \xi}{(y-\xi)(t-\xi)}\right. \\
& \left.+\frac{d}{d y} \cdot \int_{a_{k-1}}^{y} \frac{\eta(k)\left(a_{2 i}-b^{\mu} d \xi\right.}{(t-\xi)}\right)
\end{aligned}
$$

Substituting $\mathrm{k}=1,3,5 \ldots \ldots \mathrm{n}-1$.in equation (49) we will get $\mathrm{n} / 2$ simultaneous Fredholm Integral equations of the second kind. With the help of these $\mathrm{n} / 2$ simultaneous equations we can calculate $\bar{\Psi}_{1}(y)_{0} \bar{\Psi}_{a}(y)_{0 \ldots \ldots} \bar{\Psi}_{n-1}(y)$ and then the values of $\Psi_{1}(t), \Psi_{a}(t), \ldots, \Psi_{n-1}(t)$ can be determined .After all these calculations we can compute the coefficient $D_{m}$ with the help of equation (33).

\section{REFERENCES}

[1] R. Askey, "Dual equation and classical orthogonal polynomials”, J. Math.Appl. vol. 24, pp. 677-685, 1968.

[2] A.P. Dwivedi, and T. N. Trivedi, "Quadruple series equation involving Jacobi polynomials”, Proc. Nat. Acad. Sci. India vol.42 (A), pp. 203-208,1972.

[3] A. P. Dwivedi and R. Singh, "Quadruple series equations involving Jacobi Polynomials”, Journal P. A. S. Jaunpur vol.4,pp. 1-8,1963.

[4] A.P. Dwivedi, R.G. Gupta, and P. Gupta, "Certain four series equation involving Jacobi polynomials”, Actaciencia indica,vol.4, pp. 19-21,1984.

[5] A.P. Dwivedi, S.U. Siddiqui, J. Chandel and P. Bajpai, "Six Series Equations Involving Jacobi Polynomials of Different Indices, Acta, CierciaIndica, vol. 2, pp. 243-247, 2004.
[6] A.P Dwivedi , T. Siddiqui, "Seven Series Equations Involving Jacobi Polynomials of Different Indices, Acta, CierciaIndica, vol. 2, pp. 387-392, 1998.

[7] A. P. Dwivedi, S.U. Siddiqui, J. Chandel and S. Singh "System of n Series equations Involving Jacobi Polynomials, Ganita, vol. 55 (i), pp. 33-40, 2004.

[8] K. Narain, "Certain Quadruple series equations” Scirj, vol. 2, pp. 6-30, 2013.

[9] G. Szego, “Orthogonal polynomials”, Amer, Math. Soc. Collog. Pub.

[10] I. Shukla, A.P. ,Dwivedi and V. Upadhyay, “ N-fourier Series equations involving Heat polynomials” , Int.J.Sc.Res, vol.5, pp.1435-1441, 2016. 